\title{
Bariatric Surgery: The Impact of Socioeconomic Factors and Indigenous Status
}

\section{Background}

- Prevalence of obesity much higher in Indigenous populations at $37.8 \%$ vs $22.6 \%$ for non-Indigenous

- Rate of diabetes is two times higher and manifests at lower age and lower body mass index (BMI)

- Bariatric surgery is the only proven modality to treat severe obesity $(\mathrm{BMI} \geq 35)$

- Bariatric surgery rarely performed on Indigenous people with Bariatric Outcomes Longitudinal Database (BOLD) study finding only $0.46 \%$ of bariatric surgery done on Indigenous people in the US.

\section{Objectives}

- To determine socioeconomic factors that influence access to bariatric treatment and surgery for Indigenous Canadians

\section{Methods}

- Retrospective review of all patients of the Edmonton Adult Bariatric Specialty Clinic who were discharged from clinic care in 2016

- Clinical data included BMI, comorbidities, surgical details, and complications

- Socioeconomic data included age, sex, education, marital status, occupation, alcohol use, smoking, Indigenous status, and estimated incomes

- The Data Integration, Measurement \& Reporting (DIMR) database was used to acquire Indigenous statuses and estimated incomes

- Incomes were estimated from postal codes and Statistics Canada data

\section{Results}

Table 1. Patient demographics, mean $\pm \mathrm{sd}$ or $\mathrm{n}(\%)$

\begin{tabular}{|l|r|r|r|} 
& $\begin{array}{r}\text { Study } \\
\text { population }\end{array}$ & $\begin{array}{r}\text { Completed } \\
\text { program }\end{array}$ & $\begin{array}{r}\text { Non- } \\
\text { completion }\end{array}$ \\
\hline Mean age (years) & $44.8 \pm 11.4$ & $44.7 \pm 10.1$ & $44.9 \pm 11.9$ \\
$\mathrm{n}=235$ & 546 \\
\hline Female & $569(72.9)$ & $193(82.1)$ & $376(68.9)$ \\
\hline Initial BMI $\left(\mathrm{kg} / \mathrm{m}^{2}\right)$ & $47.8 \pm 8.7$ & $48.2 \pm 9.6$ & $48.4 \pm 9.3$ \\
\hline Comorbidities & & & \\
\hline Diabetes & $240(30.8)$ & $72(30.6)$ & $168(30.8)$ \\
\hline Hypertension & $360(46.1)$ & $111(47.2)$ & $249(45.7)$ \\
\hline Dyslipidemia & $206(26.4)$ & $73(31.1)$ & $133(24.5)$ \\
\hline Coronary artery disease & $46(5.9)$ & $12(5.1)$ & $34(6.2)$ \\
\hline Osteoarthritis & $252(32.3)$ & $63(26.8)$ & $189(34.7)$ \\
\hline Obstructive sleep apnea & $367(47.1)$ & $114(48.5)$ & $253(46.4)$ \\
\hline Asthma & $123(15.8)$ & $27(11.5)$ & $96(17.6)$ \\
\hline Anxiety & $154(19.7)$ & $42(17.9)$ & $112(20.6)$ \\
\hline Depression & $352(45.1)$ & $104(44.3)$ & $248(45.5)$ \\
\hline Hypothyroidism & $146(18.9)$ & $54(23.0)$ & $92(16.9)$ \\
\hline Non-alcoholic fatty liver disease & $100(12.8)$ & $12(5.1)$ & $88(16.2)$
\end{tabular}

Table 2. Demographics of Indigenous cohort, mean $\pm \mathrm{sd}$, median (IQR) or $n(\%)$

\begin{tabular}{l|}
\hline Mean age (years) \\
\hline Female \\
\hline $\mathrm{BMI}\left(\mathrm{kg} / \mathrm{m}^{2}\right)$ \\
\hline Driving distance from bariatric clinic $(\mathrm{km})$ \\
\hline Education \\
\hline Marital status \\
\hline Occupation \\
\hline Estimated Income (CAD) \\
\hline
\end{tabular}

$+$

\section{$47.0+5.3$}

$5(71.4)$

$58.8 \pm 11.7$

$6.8(2.8-247.6)$

Secondary $(57.1 \%)$

Single $(71.4 \%)$

Unemployed (100\%)

$55739 \pm 23853$ $\square$ Not a candidate for

bariatric surgery

- Ongoing

medical/psychiatric issue

$\square$ Patient decision

$\square$ Poor compliance

$\square$ Completed program

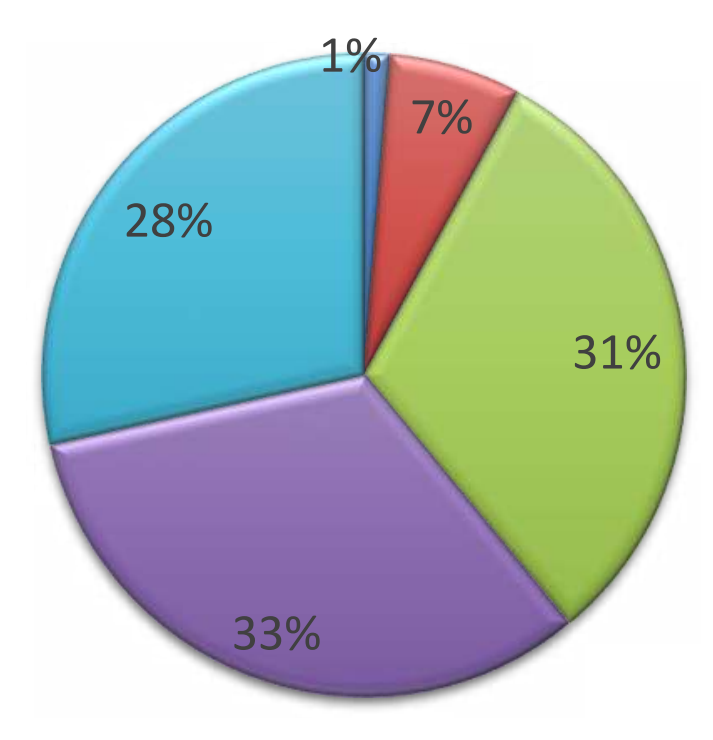

Table 3. Socioeconomic factors, mean \pm sd or median (IQR) or $n(\%)$

\begin{tabular}{|l|r|r|r|} 
& $\begin{array}{r}\text { Study } \\
\text { population }\end{array}$ & $\begin{array}{r}\text { Completed } \\
\text { program }\end{array}$ & $\begin{array}{r}\text { Non- } \\
\text { completion }\end{array}$ \\
\hline Distance to bariatric centre & $17.7(10.7-$ & $21.4(12.7-$ & $16.0(9.8-$ \\
$(\mathrm{km})$ & $72.3)$ & $213.7)$ & $46.6)$ \\
\hline Estimated income (CAD) & $66231 \pm 25769$ & $69599 \pm 25041$ & $64838 \pm 25849$ \\
\hline Indigenous status & $7(0.94)$ & $0(0)$ & $7(1.3)$ \\
\hline Smoker & $105(13.6)$ & $13(5.6)$ & $92(17.0)$ \\
\hline Alcohol abuse & $10(1.3)$ & $0(0)$ & $10(1.86)$
\end{tabular}

Table 4. Education, n (\%)

\begin{tabular}{|l|r|r|r|}
\hline & Study population & Completed program & Non-completion \\
\hline Primary & $17(2.2)$ & $3(1.3)$ & $14(2.6)$ \\
\hline Secondary & $264(34.7)$ & $72(31.4)$ & $192(36.1)$ \\
\hline College & $307(40.3)$ & $96(41.9)$ & $211(39.7)$ \\
\hline University & $173(22.7)$ & $58(25.3)$ & $115(21.6)$
\end{tabular}

Table 5. Marital status, n (\%)

\begin{tabular}{|l|r|r|r|} 
& Study population & Completed program & Non-completion \\
\hline Single & $186(24.1)$ & $38(16.5)$ & $148(27.4)$ \\
\hline Widowed & $12(1.6)$ & $4(1.7)$ & $8(1.5)$ \\
\hline Separated & $89(11.5)$ & $23(10)$ & $66(12.2)$ \\
\hline Common-law & $86(11.2)$ & $19(8.3)$ & $67(12.4)$ \\
\hline Married & $398(51.6)$ & $146(63.5)$ & $252(46.6)$
\end{tabular}

Table 6. Occupation, n (\%)

\begin{tabular}{|l|r|r|r|}
\hline & Study population & Completed program & Non-completion \\
\hline Unemployed & $155(20.3)$ & $38(16.6)$ & $117(22.0)$ \\
\hline Student & $9(1.2)$ & $3(1.3)$ & $6(1.1)$ \\
\hline Labour & $38(5.0)$ & $9(3.9)$ & $29(5.4)$ \\
\hline Intermediate & $173(22.7)$ & $50(21.8)$ & $123(23.1)$ \\
\hline Skilled trades & $205(26.9)$ & $74(32.3)$ & $131(24.6)$ \\
\hline Management & $108(14.1)$ & $40(17.5)$ & $68(12.8)$ \\
\hline Professional & $74(9.7)$ & $15(6.6)$ & $59(11.1)$
\end{tabular}

Table 7. Factors predictive of completion of bariatric surgery

\begin{tabular}{|l|r|r|}
\hline Socioeconomic Factor & Odds Ratio (95\% Cl) & $p>z$ \\
\hline Married & $2.04(1.2-3.5)$ & 0.008 \\
\hline Non-smoker & $0.30(0.14-0.65)$ & 0.002 \\
\hline Female & $0.33(0.19-0.56)$ & $<0.001$ \\
\hline
\end{tabular}

\section{Discussion}

- 781 patients in study with 235 completing the bariatric surgery program.

- Only seven Indigenous patients seen in clinic with none completing bariatric surgery program.

- Factors predictive of completion of bariatric program were being married, females and non-smokers.

- Other socioeconomic factors such as education, income, and occupation were not predictive.

- Inequities to necessary health care are unacceptable and contribute to gaps in health status between Indigenous and non-Indigenous people.
- Improving access to bariatric care for Indigenous populations include improving patient-provider communication by understanding sociocultural and pollical contexts.

- This includes understanding Indigenous customs, language, the historical reasons for mistrust, the importance of Indigenous family structures and allowing for Indigenous medicine in treatment.

- Limitations to this study include: retrospective nature, potentially overinflated education and occupations due to self-reporting, inaccuracy of income estimations, and only capturing patients seen in clinic.

\section{Conclusions}

- Despite an alarming rate of obesity in Indigenous populations, few are treated in specialized bariatric treatment centres or with bariatric surgery.

- More effort should be aimed at removing barriers to bariatric care and improving treatment to underserved populations. 